\title{
Technè
}

La science au service de l'histoire de l'art et de la préservation des biens culturels

40 | 2014

Thérapéia. Polychromie et restauration de la sculpture dans l'Antiquité

\section{Réparer les idoles, entretenir les lieux de culte. L'économie de la restauration dans les sanctuaires du monde grec}

Véronique Chankowski

\section{CpenEdition}

Journals

Édition électronique

URL : https://journals.openedition.org/techne/3346

DOI : $10.4000 /$ techne.3346

ISSN : 2534-5168

Éditeur

C2RMF

\section{Édition imprimée}

Date de publication : 26 novembre 2014

Pagination : $42-45$

ISBN : 978-2-7118-6218-4

ISSN : 1254-7867

Référence électronique

Véronique Chankowski, « Réparer les idoles, entretenir les lieux de culte. L'économie de la restauration dans les sanctuaires du monde grec », Technè [En ligne], 40 | 2014, mis en ligne le 24 juillet 2020, consulté le 08 février 2022. URL : http://journals.openedition.org/techne/3346 ; DOI : https://doi.org/ 10.4000/techne.3346

\section{(c) (1) $\Theta$}

La revue Technè. La science au service de l'histoire de l'art et de la préservation des biens culturels est mise à disposition selon les termes de la Licence Creative Commons Attribution - Pas d'Utilisation Commerciale - Pas de Modification 4.0 International. 


\section{Véronique Chankowski \\ Réparer les idoles, entretenir les lieux de culte. L'économie de la restauration dans les sanctuaires du monde grec}

Repairing idols, maintaining places of worship. The restoration budget for sanctuaries in the Greek world

Résumé. La restauration des cuvres d'art et tout particulièrement celle des statues dans les lieux de culte du monde grec antique peut être perçue à travers les sources littéraires et épigraphiques qui laissent entrevoir l'implication d'artisans spécialisés, mais également l'implication financière et institutionnelle des organismes civiques concernant la remise en état des sanctuaires et des éléments du culte. On envisage ici plusieurs documents, principalement aux époques classique et hellénistique (IV $I^{e}{ }^{e r}$ siècles av. J.-C.).

Mots-clés. Dorure, restauration, statue, artisans, contrat, souscription, évergétisme, Délos.
Abstract. The restoration of artworks, especially that of statues in places of worship in the Ancient Greek world, can be perceived through literary and epigraphic sources that provide insight not only into the role of specialized craftsmen but also the financial and institutional involvement of civic entities concerning the renovation of sanctuaries and cult objects. Several documents are considered here, mainly dating from the Classical and Hellenistic periods (4th-1st century $B C$ ).

Keywords. Gilding, restoration, statue, craftsmen, contract, subscription, euergetism, Delos.
Témoignages d'une histoire qui remontait la plupart du temps aux mythes fondateurs des cités, des objets précieux et symboliques étaient souvent conservés avec soin par les communautés grecques de l'Antiquité. Ils s'apparentent à des reliques censées témoigner, par l'objectivation et la matérialité des vestiges, de la réalité d'épisodes racontés dans les mythes, lesquels, considérés comme vécus par ces communautés, sont devenus fondateurs de la construction d'une identité commune. Généralement liés à la personne des héros fondateurs, ces objets sont souvent partie intégrante des cultes civiques. Il n'est pas rare qu'ils assurent une fonction de protection de la communauté civique, à l'instar du fameux palladion, statue de Pallas-Athéna qui, selon la légende, garantissait à la cité qui la détenait (Troie, puis Rome) la protection des dieux ${ }^{1}$.

La conservation des reliques et, plus largement, l'entretien des statues de culte qui n'avaient pas toutes ce statut de relique, sont donc constitutifs de l'activité menée dans les sanctuaires. Les gestes de la conservation et de la restauration entretiennent pourtant une relation ambiguë à l'objet original confié à la communauté civique par les dieux ou par les héros des temps anciens. Dans un passage de la Vie de Thésée $(23,1)$, Plutarque rapporte que les Athéniens avaient, jusqu'à la fin du IV ${ }^{\mathrm{e}}$ siècle av. J.-C., veillé à l'entretien d'un navire bien réel sur lequel le héros Thésée aurait, d'après la légende qui situe cet épisode dans les temps mythiques du premier développement de la cité athénienne, navigué depuis la Crète jusqu'en Attique en ramenant sains et saufs les jeunes gens d'Athènes qu'il avait sauvés du Minotaure ${ }^{2}$. Il ajoute que cet exemple était utilisé par les philosophes dans leurs débats sur la nature des êtres:
«Le navire sur lequel il avait fait la traversée avec les jeunes gens et était revenu sain et sauf était un navire à trente rameurs que les Athéniens conservèrent jusqu'au temps de Démétrios de Phalère ${ }^{3}$. Ils en retiraient les planches trop vieilles et y substituaient des planches solides qu'ils ajustaient avec les autres. Aussi les philosophes, quand ils disputent sur ce qu'ils appellent "l'argument de croissance", citent ce vaisseau comme un exemple controversé, les uns prétendant qu'il est resté le même et les autres le niant ${ }^{4}$. »

La conservation et la restauration transforment l'objet initial tout en lui assurant une pérennité : c'est bien de cette ambiguïté dont devaient s'accommoder, dans le monde grec, les gestionnaires de l'équipement cultuel. Plusieurs grands sanctuaires du monde grec ont livré une documentation abondante de nature purement administrative : il s'agit de la comptabilité, gravée sur pierre, tenue annuellement par des magistrats ou des préposés à la gestion des sanctuaires et validée officiellement par les vérificateurs des comptes de la cité. Nous disposons ainsi, entre le $\mathrm{V}^{\mathrm{e}}$ et le $\mathrm{I}^{\mathrm{er}}$ siècle av. J.-C., de plusieurs centaines de comptes annuels, très détaillés, qui énumèrent les dépenses diverses générées par la construction et l'administration des sanctuaires : construction et réparation de bâtiments et d'objets, achats de matériaux et de denrées destinés aux fêtes et aux banquets, salaires d'artisans et frais de personnel, mais aussi gestion des capitaux sous la forme de prêts à intérêts à destination des particuliers, et perception de taxes diverses. C'est bien d'économie et de gestion dont parle, aussi, la documentation du domaine sacré.

Véronique Chankowski, professeur d'histoire grecque, Université Lyon 2, UMR 5189 HiSoMA (veronique.chankowski@mom.fr). 
Parmi les multiples perspectives d'étude offertes par les comptes des sanctuaires, il en est une qui se place naturellement à la frontière entre l'épigraphie, l'histoire de l'art et l'histoire des pratiques religieuses : elle concerne le traitement accordé aux statues d'après les opérations de conservation et de restauration mentionnées dans les comptes gravés ${ }^{5}$. Le corpus épigraphique est assez maigre, car ce sont surtout les consécrations de nouvelles statues financées par des évergètes ou par les cités qui ont donné lieu à l'inscription de dédicaces. Seuls les comptes des sanctuaires fournissent quelques informations sur des opérations d'entretien et de restauration des bâtiments cultuels et des statues.

\section{Recruter les artisans : le vocabulaire de la restauration dans les contrats}

Une partie des opérations d'entretien était réalisée, dans les sanctuaires, par du personnel affecté, ou appointé occasionnellement. C'est le cas de travaux de nettoyage des lieux de culte, qui apparaissent fréquemment dans les comptes. La kosmèsis (ou épikosmèsis) de la statue, étendue parfois à l'ensemble du sanctuaire, désigne le nettoyage et l'ornementation qui marquaient les jours de fête. Un prêtre d'Apollon Zôster, en Attique, est ainsi honoré au IV siècle av. J.-C. par le dème d'Halai pour avoir « remis en état le sanctuaire et orné la

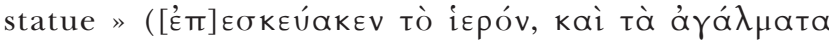

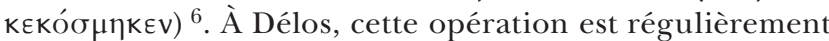
mentionnée à propos du culte d'Héra et confiée à des salariées, chargées de faire rituellement un nettoyage annuel du sanctuaire ${ }^{7}$. Mais son emploi dans d'autres contextes, impliquant des artisans, fait dire à J. Marcadé que le terme a un double sens, « un sens précis appartenant à la langue religieuse et un sens large appartenant à la langue des artisans ${ }^{8}$ ».

En effet, les soins requis par l'état des statues nécessitaient parfois d'avoir recours à des artisans spécialisés, sous la forme d'un contrat d'adjudication proposé par les sanctuaires au mieux disant. Des artisans de différentes régions du monde grec pouvaient ainsi proposer leurs services ${ }^{9}$. Il n'est pas rare qu'à l'issue de son séjour, l'artisan soit remercié de ses bons services, non seulement par le paiement du contrat, mais également par un décret honorifique lui accordant quelques privilèges supplémentaires. Ainsi, au début du $\mathrm{III}^{\mathrm{e}}$ siècle av. J.-C., un décret de la cité de Délos honore des titres d'évergète («bienfaiteur») et de proxène (« hôte public») le sculpteur athénien Télèsinos qui, ayant pris en adjudication la fabrication de deux statues, l'une d'Asklépios, en bronze, et l'autre de la reine Stratonice, en marbre, a non seulement réalisé ces deux œuvres, mais a également employé une partie de son temps et son art à restaurer gratuitement des statues du sanctuaire d'Apollon qui en avaient besoin : kaì tà



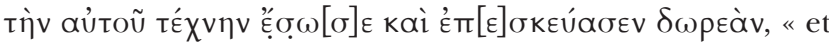
pour les statues du sanctuaire qui avaient besoin d'une réparation, il les a sauvées grâce à son art et les a réparées gratuitement ${ }^{10}$. » La restauration (désignée ici sous le vocable de

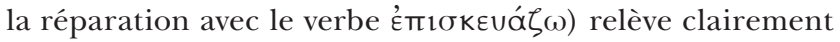
d'une technè propre à l'artisan ${ }^{11}$.

Un autre artisan, Ôphélion, a également travaillé à Délos sur des effigies divines au milieu du III $^{\mathrm{e}}$ siècle av. J.-C. Il semble spécialisé dans la décoration des statues et emporte plusieurs contrats d'adjudication pour le sanctuaire délien ${ }^{12}$. Il y travaille donc à plusieurs reprises, d'abord à la dorure et à la décoration de la statue d'Hécate (IG XI, 2, 287, A, 1. 114-115 :

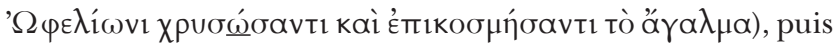
à la décoration de la statue d'Artémis « en l'île » (ID 290, 1.

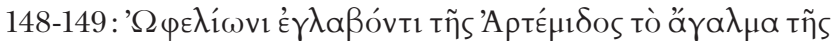

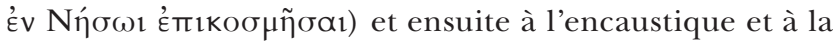
décoration de celle de l'Aphrodision consacré par Stèsiléôs

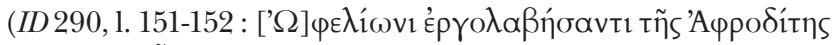

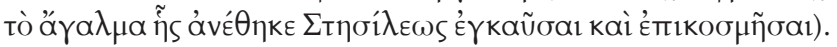
Enfin, la même année, il prend en adjudication un travail sur trois statues du Pythion (ID 290, 1. 229-231, 233-235 et 241244) : il s'agit cette fois d'un travail de restauration puisque le contrat, dont le compte reprend sans doute l'essentiel des rubriques, prévoit qu'il devra «peindre à l'encaustique

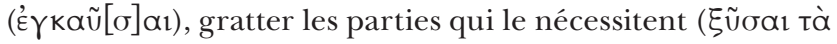

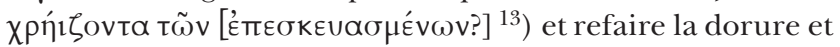

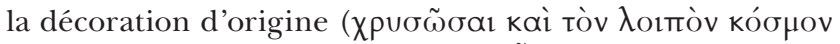

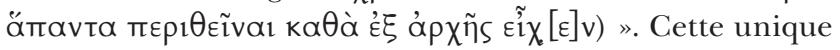
mention des statues du Pythion ne permet pas de savoir quelles divinités elles représentaient (peut-être la triade apollinienne), mais la volonté des Déliens, en 246, de redonner aux statues leur aspect originel, sans doute celui de leur création à l'initiative des Athéniens dans la seconde moitié du IV ${ }^{\mathrm{e}}$ siècle, est intéressante. Cette remise en état n'était probablement pas la première, à l'image des réparations multiples réalisées par les Athéniens sur le bateau de Thésée pour lui conserver son apparence première.

La dorure des statues (chrysôsis) fait donc partie des opérations de kosmèsis organisées régulièrement dans les sanctuaires. À Délos, d'autres statues font l'objet de travaux de dorure : les comptes mentionnent des interventions d'artisans pour des opérations de kosmèsis qui, parfois, comportent explicitement un travail de dorure à la feuille d'or. Ainsi, en 218, l'artisan Apollodôros travaille sur une statue d'Aphrodite et se fait fournir par le sanctuaire des feuilles d'or, $\pi \dot{\epsilon} \tau \alpha[\lambda \alpha]$ $(I D 354,1.77)^{14}$. Mais on ignore souvent s'il s'agit d'une remise en état ou d'une première dorure, réalisée longtemps après l'installation de la statue de culte.

Une autre opération d'entretien, la ganôsis, est mentionnée dans les comptes de Délos et dans une inscription du Ptoion en Béotie : il s'agirait, d'après des descriptions fournies par Vitruve et Plutarque pour les statues romaines, d'une opération de glaçage ou de vernis à la cire appliqué par les restaurateurs, principalement sur des marbres peints ${ }^{15}$. Mais comme le montrent les cas déliens où des artisans interviennent pour


statues d'une autre nature : le xoanon en bois d'Aphrodite ou la statue chryséléphantine de Tektaios et Angélion ${ }^{16}$.

Le vocabulaire de la kosmèsis contient donc une certaine polyvalence, s'appliquant tout aussi bien encore à l'habillement 
des idoles de bois ou des acrolithes avec des tissus colorés et coûteux : à Délos, de la pourpre pour Létô (ID 203, A, 1. 73), du linge fin pour Héra (IG XI 2, 154, A, 1. 22), de la pourpre brochée d'or pour Artémis (ID 1442, B, 1. 54-56).

\section{Organiser la dépense : les actes d'évergétisme dans la restauration}

Dans le cas des grands sanctuaires, la caisse sacrée est en mesure d'assumer les réparations régulières nécessaires à l'entretien des bâtiments et des objets de culte. Il revient aux citoyens chargés d'administrer les biens sacrés de veiller à leur conservation, parmi d'autres tâches diverses ${ }^{17}$. À l'époque

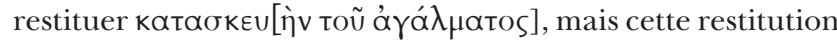
est manifestement trop courte pour la longueur de la ligne conservée $^{19}$.

Au cours de la période hellénistique, nombreux sont les témoignages épigraphiques de souscriptions qui, dans plusieurs cas, concernent un projet de réfection ou de restauration d'édifices religieux pour les cités. La restauration du temple d'Asclépios à Orchomène, entre 250 et 220 av. J.-C., se fait sous la responsabilité de commissaires à la restauration

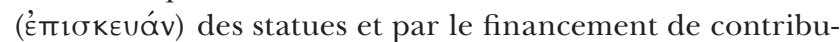
teurs ${ }^{20}$. À Histiée, une souscription publique permet, à la fin du $\mathrm{II}^{\mathrm{e}}$ siècle av. J.-C., la restauration, ou plus exactement le


et la fabrication de sa statue (SEG XXXIV, 909). À Olymos, c'est encore la générosité des souscripteurs qui rend possible la restauration du sanctuaire de Létô dans la seconde moitié du II ${ }^{\mathrm{e}}$ siècle av. J.-C., avec peut-être une mention de la statue de culte ${ }^{21}$. Dans d'autres cas, c'est explicitement la parure de la statue qui est mentionnée : à Lindos sur l'île de Rhodes, probablement à la suite de l'incendie qui détruisit le temple d'Athéna en 329/1, des contributeurs se cotisent pour reconstituer la parure et la vaisselle d'Athéna (Lindos II, 51).

Des bienfaiteurs individuels apparaissent également comme les financeurs d'opérations impliquant parfois la dorure de la statue, sans que l'on puisse savoir s'il s'agit d'une réfection ou bien de la transformation d'une statue à laquelle la dorure doit donner une nouvelle apparence. À Tégée, au début de l'époque impériale, est conservée la dédicace faite au nom de son fils par un certain Philocratès fils de Damonikos, qui « a consacré l'autel et a fait dorer la statue d'Apollon»

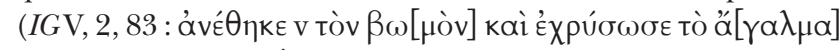
тои̃ 'Aтó $\lambda \lambda \omega v$ [os]). À Délos encore, à la fin du II ${ }^{\mathrm{e}}$ siècle av. J.-C., deux épimélètes athéniens de l'île signalent, dans une dédicace à Aphrodite, avoir restauré à leurs frais la statue (tò

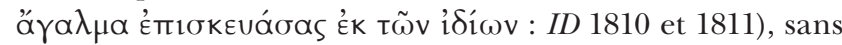
que soit précisée la nature de l'opération.

La restauration des édifices, et plus particulièrement celle des statues de culte, fait bel et bien partie des préoccupations des cités grecques, qui mobilisent des magistrats et des revenus à cet effet ${ }^{22}$. Tout en s'insérant dans des dispositifs et des processus rituels, le traitement de la statue cultuelle révèle surtout l'intervention de spécialistes qui sont en mesure de mettre en œuvre des techniques artisanales faisant leur réputation. La préoccupation des commanditaires semble le plus souvent marquée par la volonté de redonner à l'objet cultuel son apparence d'origine, mais certaines opérations de dorure pourraient avoir également offert une nouvelle apparence à des statues anciennes. 
Notes

Liste des sigles et abréviations de collections

C.U.F. : Collection des Universités de France, Paris.

FGH : F. Jacoby et al., Die Fragmente der griechischen Historiker, Brill.

ID : Corpus des Inscriptions de Délos, Paris.

$I G$ : Inscriptiones Graecae, Berlin.

SEG : Supplementum Epigraphicum Graecum, Amsterdam

ThesCRA : Thesaurus Cultus et Rituum Antiquorum, J. P. Getty Museum, Los Angeles.

1. Apollodore, Épitomé V, 10.

2. Sur l'interprétation de ce passage dans l'organisation des fêtes déliennes, voir Chankowski, 2008, p. 88-89.

3. Homme d'État athénien, Démétrios de Phalère fut gouverneur d'Athènes sous l'autorité macédonienne entre 317 et 307 av. J.-C.

4. Trad. R. Flacelière, C.U.F.

5. Pour une perspective plus large sur les rites et activités relatives aux images cultuelles, on se reportera au volume II, 5 du ThesCRA.

6. SEG 42, 112, 1. 3-4 (D. Gill, Greek Cult Tables, New York, 1991, p. 48-50 n 19).

7. Voir Bruneau, 1970, p. 253-254, en opposition à l'opinion exprimée par Marcadé, 1969, p. 98-99, qui comprend la kosmèsis comme une opération consistant à laver et à badigeonner la statue. Pour Bruneau, l'opération est confiée à des

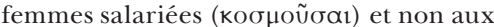
prêtresses d'Héra comme le pensait Roussel, 1916 , p. 244, n. 6.

8. Marcadé, 1969, p. 101 et, plus largement, p. 95-102, pour l'emploi des termes grecs de la restauration dans les comptes déliens.

9. Sur l'activité des artisans dans les sanctuaires grecs, voir Feyel, 2006. L'ouvrage est fondé sur les corpus épigraphiques des comptes de construction de Delphes, Délos,
Éleusis et Épidaure, mais ne traite pas la documentation d'Asie mineure.

10. IG XI 4, 514, 1. 13-16. Ce témoignage n'est pas répertorié dans la liste des artisans fournie par l'ouvrage Feyel, 2006, qui ne prend pas en compte les décrets de la cité de Délos.

11. Ce même Télésinos, athénien, est probablement celui que mentionne Philochore (FGH I, p. 414, n ${ }^{\circ} 185$ ) comme sculpteur des statues colossales de Poséidon et Amphitrite à Ténos. Voir Muller-Dufeu, 2002, p. 833.

12. Feyel, 2006, p. 289, DÉL 562

13. La restauration de ce passage lacunaire, telle qu'elle a été proposée par l'éditeur de cette inscription, F. Durrbach, dit littéralement « gratter les réparations antérieures qui le nécessitent " mais le terme

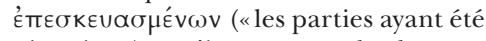
réparées") ne s'impose pas absolument ici. Il pourrait tout aussi bien s'agir de gratter les parties à redorer.

14. Lecture de J. Tréheux : $\pi \varepsilon ́ \tau \alpha[\lambda \alpha]$ au


n. 6 .

15. Voir dans ce volume, p. 69 Plutarque, Moralia IV, Quaest. Rom. 98 D (287), pour expliquer la raison pour laquelle les censeurs romains s'occupent en priorité de la ganôsis dès leur entrée en charge : 'H $\delta$ غ̇

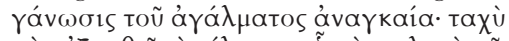

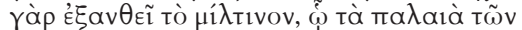

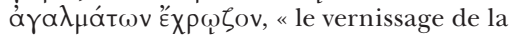
statue est absolument nécessaire, car le pigment rouge avec lequel les statues anciennes étaient colorées perd rapidement de sa fraîcheur».

16. Holleaux M., BCH 14 (1890), p. 181187 ; Marcadé, 1969, p. 102-103. Inscriptions mentionnant la ganôsis : IG VII, 4149, 1 . 18 (Ptoion) ; IG XI 2, 144, B, 1. 5 ; IG XI 2, 228, l. 12 ; ID 461, Ab, 1. 35 (Délos).

17. Voir par exemple, pour Délos, la description des fonctions incombant aux hiéropes dans Vial, 1984, p. 216-231.

18. Migeotte, 2014, p. 293 ; Migeotte, 1992, p. 307.
19. Migeotte, 1992, p. 24-25.

20. Preuner E., Athenische Mitteilungen 49 (1924), p. 125-134 ; Migeotte, 1992, p. $70-75$.

21. Blümel W., Epigraphica Anatolica 13 (1989), p. 7-9 ; Migeotte, 1992, p. 236-240.

22. La question du coût des métaux précieux et des produits utilisés pour l'entretien et pour la polychromie des statues et des édifices est complexe : Chankowski V., "Un marché de la couleur? Quelques considérations sur les enjeux économiques de la polychromie " dans Jockey Ph. (dir.), Les arts de la couleur en Grèce ancienne, Actes du colloque d'Athènes, 23-25 avril 2009, Athènes, BCH Suppl., à paraître.

\section{Bibliographie}

Bruneau Ph., 1970, Recherches sur les cultes de Délos à l'époque hellénistique et à l'époque impériale, BEFAR, 217, Paris.

Chankowski V., 2008, Athènes et Délos à l'époque classique. Recherches sur l'administration du sanctuaire d'Apollon délien, BEFAR, 331, Athènes.

Feyel Chr., 2006, Les artisans dans les sanctuaires grecs aux époques classique et hellénistique à travers la documentation financière en Grèce, BEFAR, 318, Athènes.

Marcadé J., 1969, Au Musée de Délos. Étude sur la sculpture hellénistique en ronde-bosse découverte dans l'île, BEFAR, 215, Paris.

Muller-Dufeu M., 2002, La sculpture grecque. Sources littéraires et épigraphiques, éditions de l'École nationale supérieure des Beaux-Arts, Paris.

Migeotte L., 2014, Les finances des cités grecques, Les Belles Lettres, Paris.

Migeotte L., 1992, Les souscriptions publiques dans les cités grecques, Droz, Genève.

Roussel P., 1916, Délos colonie athénienne, BEFAR, 111, Paris

Vial Cl., 1984, Délos Indépendante. Étude d'une communauté civique et de ses institutions, $B C H$, Suppl. X. 